\title{
Avaliação do tratamento antirretroviral de pessoas convivendo com HIV/aids que participam de um grupo de adesão
}

\author{
Evaluation of antiretroviral treatment of people living with HIV/aids participating \\ in an adherence group
}

Hilana Francisca Nascimento Silva ${ }^{1}$ (D), Carla Solange de Melo Escórcio Dourado ${ }^{2}$ (D), Hengrid Graciely Nascimento Silva ${ }^{3}$ (D), Hisamara Fernanda Nascimento Silva ${ }^{4}$ (D)

\begin{abstract}
RESUMO
Modelo de estudo: Trata-se de um estudo do tipo observacional, descritivo e transversal. Objetivo: Avaliar o comportamento de adesão ao tratamento antirretroviral de pessoas convivendo com HIV/aids que participam de um Grupo de Adesão. Métodos: O questionário foi respondido por 15 pacientes maiores de 18 anos, independente do sexo, com diagnóstico confirmado de AIDS que participam de um Grupo de Adesão em um serviço de dispensação de antirretrovirais, e que após receberem informações pertinentes ao estudo, aceitaram participar voluntariamente, por meio da assinatura do Termo de Consentimento Livre e Esclarecido. Resultado: A maioria dos participantes era do sexo masculino, acima de 40 anos $(73,3 \%)$ e 26,6\% eram economicamente ativos. Quanto à sexualidade, $80 \%$ dos entrevistados declararam ser heterossexual. Quanto à escolaridade, $60 \%$ não havia completado o ensino médio. O tempo de diagnóstico da doença predominante foi entre 10 e 16 anos, sendo que 46,6\% referiram possuir outros problemas de saúde, como toxoplasmose, herpes, tuberculose e leishmaniose. A análise do questionário de adesão mostrou que 33,3\% possuía uma boa adesão ao tratamento antirretroviral. Entre as interações droga-droga identificadas, $44,4 \%$ ocorreram entre os antirretrovirais. Conclusão: A partir da realização deste estudo foi possível concluir que $66,66 \%$ dos entrevistados relataram que em algum momento houve uma descontinuidade do tratamento, revelando uma dificuldade na manutenção do uso dos antirretrovirais em indivíduos convivendo com HIV/AIDS. No contexto da farmacoepidemiologia antirretroviral, as potenciais interações medicamentosas identificadas neste estudo foram eventos que afetam a resposta terapêutica levando a toxicidade.
\end{abstract}

Palavras-chave: Imunodeficiência; Aids; Fármacos Anti-HIV; Farmacoepidemiologia.

\begin{abstract}
Study design: This is an observational, descriptive, and cross-sectional study. Objective: Evaluating the adherence behavior to the antiretroviral treatment of people living with HIV/Aids who participate in an Adherence Group. Methods: The questionnaire was answered by 15 patients older than 18 years of age, regardless of sex, with a confirmed diagnosis of AIDS participating in an Adherence Group at an antiretroviral dispensing service, who, after receiving information related to the study, agreed to participate voluntarily, by singing the Written Informed Consent Form. Results: The majority of the participants were male, over 40 years old $(73.3 \%)$ and $26.6 \%$ were economically active. Regarding sexuality, $80 \%$ of the interviewees stated that they were heterosexual. As for schooling, $60 \%$ had not finished high school. The time of diagnosis of the disease predominant was between 10 and 16 years, and $46.6 \%$ reported having other health problems, like toxoplasmosis, herpes, tuberculosis, and leishmaniasis. The analysis of the adherence questionnaire showed that $33.3 \%$ had good adherence to antiretroviral treatment. Among the drugdrug interactions identified, $44.4 \%$ occurred among antirretrovirals. Conclusion: Based on this study, it was possible to conclude that $66,66 \%$ at some point there was a discontinuation of treatment, revealing a difficulty in maintaining antiretroviral use in individuals living with HIV/aids. In the scenario of antiretroviral pharmacoepidemiology, the potential drug interactions identified in this study were events that affect the therapeutic response leading to toxicity.
\end{abstract}

Keywords: Immunodeficiency; Aids; Anti-HIV Drugs; Pharmacoepidemiology.

1. Especialização em Gestão Hospitalar e Qualidade em Serviços de Saúde. Universidade Federal do Piauí (UFPI), Teresina (PI), Brasil.

2. Doutora em Programa de Pós-Graduação em Biotecnologia. UFPI, Teresina (PI), Brasil

3. Mestrado em Ciências e Saúde. Universidade Estadual do Piauí (UESPI), Teresina (PI), Brasil.

4. Graduação em Odontologia. Faculdade Facid DeVry, Teresina (PI), Brasil

$\bowtie$ Hilana Francisca Nascimento Silva. Rua: Rufino Costa e Sousa, 513 - Parque Piauí. CEP: 65631-270. Timon (MA), Brasil. hilanafns@hotmail.com | Recebido: 12/01/2018 | Aprovado: 24/04/2019 


\section{INTRODUÇÃO}

$A_{e}^{s}$ Síndrome da Imunodeficiência Humana (AIDS) é a manifestação clínica avançada decorrente de um quadro de imunodeficiência causado pelo HIV $^{1}$. A AIDS pode ser considerada uma doença crônica para a qual não há cura, mas há tratamento. A terapia antirretroviral (TARV) tem como principal objetivo retardar a evolução da doença, promover a supressão da carga viral abaixo do limiar de detecção pelo máximo de tempo possível, a restauração e a manutenção da imunidade, estimulando, deste modo, a manutenção da saúde geral e aumento da taxa de sobrevivência de indivíduos infectados².

Estudos mostraram que o sucesso da terapia antirretroviral altamente ativa (HARRT) está associado com a manutenção de uma elevada taxa de adesão do paciente em relação ao tratamento, da prevenção e gestão das interações drogas-drogas (IDDs) ${ }^{3}$. Tendo em vista a adesão como a habilidade do paciente em seguir as prescrições e recomendações a ele propostas, entendendo os benefícios que o tratamento pode trazer-lhe, uma baixa adesão pode representar uma ameaça à saúde pública, pois aumenta a probabilidade de resistência viral, proporcionando um tratamento com baixa perspectiva de controle da replicação do HIV, bem como na disseminação de um vírus multirresistente ${ }^{4}$.

Já a IDD é definida como um efeito clínico ou farmacológico resultante de uma coadministração de medicamentos, que altera a reação do paciente ao tratamento. Os agentes da terapia antirretroviral (ART) representam um dos principais grupos terapêuticos com o maior potencial para IDD. Participantes expostos ao IDD mostraram uma redução na adesão ao tratamento ${ }^{5}$.

O presente estudo teve como objetivos: (1) descrever o comportamento de adesão ao tratamento antirretroviral de pessoas convivendo com HIV/AIDS que participam de um Grupo de Adesão, (2) investigar fatores preditores da adesão à terapia antirretroviral, considerando variáveis sociodemográficas e (3) verificar as principais IDD.

\section{MATERIAL E MÉTODOS}

Trata-se de um estudo do tipo observacional, descritivo e transversal, realizado com participan- tes do Grupo de Adesão do Instituto de Doenças Tropicais Natan Portela (IDTNP), que se caracteriza por reuniões em todas as quartas, inclusive em feriados, onde são apresentados temas relevantes sobre questões de saúde em forma de palestras, grupos de discussão e dinâmicas para cerca de 20 pessoas, há 17 anos. Este grupo não possui participantes fixos, apresentando, dessa forma, uma rotatividade muito grande.

No presente estudo foram incluídos pacientes de ambos os gêneros, maiores de 18 anos, com diagnóstico confirmado de HIV/aids, participantes do Grupo Adesão do IDNTP e que concordaram em assinar o Termo de Consentimento Livre e Esclarecido (TCLE), atestando ser conscientes dos objetivos da pesquisa. Foram excluídos aqueles que não participavam do Grupo Adesão ou que desistiram de consentir com a divulgação dos dados e que optaram por desistir da pesquisa antes do completo preenchimento dos instrumentos de coleta.

A coleta de dados foi realizada com 15 pacientes entre os meses de setembro de 2016 e outubro de 2017, durante uma entrevista individual, realizada pela própria pesquisadora, em ambiente privativo, permitindo o sigilo e a confidencialidade das informações obtidas. O tempo médio da coleta de dados foi de 45 minutos.

Foi elaborado um instrumento de coleta de dados para registro das informações estruturado com as seguintes variáveis: idade, sexo, peso, altura, nível de escolaridade, estado civil, ocupação, história clínica, hábitos de vida e perfil farmacoterapêutico. Para a avaliação da adesão foi utilizada a versão validada para a língua portuguesa (Brasil) do instrumento: "Cuestionario para la Evaluación de La Adhesión al Tratamiento Antiretroviral" (CEAT-VIH) ${ }^{6}$. Trata-se de um instrumento composto por 20 itens que abordam os principais fatores que podem interferir na adesão à TARV em pacientes adultos: a história de não adesão do paciente; a relação médico-paciente; as crenças do paciente a respeito da TARV; as expectativas sobre a eficácia terapêutica; o esforço do paciente para seguir o tratamento; a avaliação da gravidade dos efeitos colaterais da TARV para o paciente; o grau de satisfação com a medicação antirretroviral e o uso de estratégias para se lembrar de tomar a medicação. Dessa forma, é 
possível avaliar o grau de adesão ao tratamento medicamentoso antirretroviral em adultos.

Depois da aplicação, fez-se o somatório dos pontos obtidos pelas respostas dos 20 itens por meio das instruções de análise descritas no manual do CEAT-VIH. A pontuação mínima possível do questionário é de 17 e, a máxima possível, de 89. O somatório das respostas dadas a cada item, posteriormente foi convertida em porcentagem para classificar os participantes nos três níveis (Tabela 1).

\section{Tabela 1}

Classificação do nível de adesão à terapia antirretroviral.

\begin{tabular}{lc}
\hline Classificação da adesão & Percentual de adesão \\
\hline Boa & Superior a $85 \%$ \\
Regular/insuficiente & Entre $50 \%$ e $84 \%$ \\
Baixa & Inferior a $50 \%$
\end{tabular}

Fonte: Autores.

Publicações regulares de periódicos nacionais e internacionais, livros citados na bibliografia e a base de dados MICROMEDEX Healthcare Series $^{7}$ foram utilizados como fonte de informação técnica. A classe terapêutica das medicações foi determinada pela classificação Anatomical Therapeutical Chemical Classification System, adotada pela Organização Mundial de Saúde (OMS) que organiza os medicamentos em diferentes grupos de acordo com o órgão ou sistema que atuam e suas propriedades químicas, terapêuticas e farmacológicas.

O estudo foi aprovado pelo Comitê de Ética em Pesquisa da Universidade Federal do Piauí sob Protocolo n. ${ }^{\circ}$ 2.139.951, atendendo as recomendações da Resolução 466/2012 do Conselho Nacional de Saúde.

\section{RESULTADOS}

Para a realização do presente estudo foram entrevistados 15 pacientes submetidos a tratamento farmacológico para HIV/AIDS pertencentes ao Grupo Adesão. Inicialmente, foi analisado o perfil sociodemográfico, no qual se verificou que $73,3 \%$ dos entrevistados pertencem ao sexo masculino, $80 \%$ caracterizaram-se como heteros- sexual, $60 \%$ possuem ensino médio incompleto, sendo importante ressaltar que $26,6 \%$ não tinham completado o ensino fundamental (Tabela 2 ).

\section{Tabela 2}

Associação entre as variáveis sociodemográficas e adesão à terapia antirretroviral satisfatória.

\begin{tabular}{|c|c|c|}
\hline \multirow[t]{2}{*}{ Variáveis } & \multicolumn{2}{|c|}{$\begin{array}{c}\text { Adesão > 85\% } \\
(n=15)\end{array}$} \\
\hline & $\mathrm{N}$ & $\%$ \\
\hline \multicolumn{3}{|l|}{ Gênero } \\
\hline Masculino & 11 & 73,3 \\
\hline Feminino & 4 & 26,7 \\
\hline \multicolumn{3}{|l|}{ Idade (anos) } \\
\hline$\leq 39$ & 4 & 26,7 \\
\hline$\geq 40$ & 11 & 73,3 \\
\hline \multicolumn{3}{|l|}{ Escolaridade } \\
\hline Ensino fundamental incompleto & 4 & 26,6 \\
\hline Ensino médio incompleto & 9 & 60,0 \\
\hline Ensino médio completo & 2 & 13,3 \\
\hline \multicolumn{3}{|c|}{ Tempo de diagnóstico de soropositividade (anos) } \\
\hline$<1$ & 1 & 6,7 \\
\hline $1-7$ & 6 & 40,0 \\
\hline$\geq 8$ & 8 & 53,3 \\
\hline \multicolumn{3}{|l|}{ Situação conjugal } \\
\hline Vive sem parceiro & 13 & 86,7 \\
\hline Vive com parceiro(a) & 2 & 13,3 \\
\hline
\end{tabular}

Fonte: Autores

A idade dos pacientes variou entre 34 e 60 anos, e a faixa etária mais prevalente foi acima de 40 anos, com $73,3 \%$ das pessoas. Quanto à situação conjugal, $86,7 \%$ se caracterizaram como solteiros.

Sobre a situação ocupacional, $26,6 \%$ das pessoas são economicamente ativas e $53,3 \%$ estão desempregadas. Verificou-se ainda que $20 \%$ dos entrevistados já se encontravam aposentados.

Em relação ao tempo de diagnóstico, foram observadas diferenças significativas no estudo. 0 menor tempo de diagnóstico entre os entrevistados foi de 5 meses e o maior de 22 anos, com valor predominante entre 10 e 16 anos de diagnóstico $(46,6 \%)$.

Verificou-se uma grande influência social no tratamento, através de relatos de atraso da 
medicação por não utilizarem no trabalho ou até mesmo em frente a familiares, já que $26,6 \%$ dos entrevistados relataram que seus familiares não sabem sobre seu diagnóstico. Além disso, o uso recreacional do álcool foi mencionado por 33,3\% dos entrevistados como uma barreira à adesão.

Quanto à percepção da própria saúde, 73,3\% dos pacientes avaliaram como sendo boa ou excelente. Entretanto, $46,6 \%$ referiram possuir outros problemas de saúde, geralmente representados por hipertensão, hipercolesterolemia, labirintite, tuberculose e leishmaniose visceral. $\mathrm{Na}$ investigação em relação à doenças oportunistas desde que foram diagnosticados, as mais citadas foram leishmaniose $(33,3 \%)$, tuberculose $(13,3 \%)$, toxoplasmose $(6,7 \%)$ e herpes simples $(6,7 \%)$. Além da medicação antirretroviral, $20 \%$ dos entrevistados referiram utilizar outros medicamentos.

No grupo em estudo, 73,3\% utilizavam dois medicamentos, ressalta-se que a maioria dos pacientes fazia uso de medicamentos compostos pela associação de dois princípios ativos em uma única forma farmacêutica, sendo neste caso, contado como um único medicamento. Em 53,3\% dos casos, os pacientes usaram uma combinação de dois inibidores da transcriptase reversa análogo do nucleosídeo (ITRN) (zidovudina, lamivudina, tenofovir ou abacavir) e um inibidor da transcriptase reversa não análogo do nucleosídeo (ITRNN) (efavirenz)transcriptase inhibitor (NNRTI) (efavirenz or nevirapine). TheA combination of 2 NRTIs and 2 protease inhibitors (PI) (lopinavir,combinação de dois ITRNs e um inibidor de protease (IP) (ritonavir ou atazanavir) foi identificado em $20 \%$ ( $n=69$ ) of the therapies.das terapias, sendo a mesma porcentagem apresentada pela combinação de dois ITRNs e um inibidor de integrasse (INI) (dolutegravir). Outros medicamentos utilizados foram sinvastatina, omeprazol, sulfametoxazol/trimetoprim, enalapril e hidroclorotiazida (Tabela 3).

\section{Tabela 3}

Associações de medicamentos mais utilizadas pelos participantes do estudo.

\begin{tabular}{lc}
\hline Associação & $\%(\mathrm{n}=15)$ \\
\hline Zidovudina / lamivudina e efavirenz & 33,3 \\
Zidovudina / lamivudina ritonavir e & 20,0 \\
atazanavir & 20,0 \\
Tenofovir / lamivudina e dolutegravir & 20,0 \\
Abacavir / lamivudina e efavirenz & 6,6 \\
Abacavir / lamivudina & \\
\hline
\end{tabular}

Fonte: Autores.

Entre os IDD identificadas, dos quais $44,4 \%$ ocorreram entre os agentes ART usados. O IDD pode resultar em mudanças na concentração de um dos medicamentos $(44,4 \%)$, ou manifestações clínicas como hepatotoxicidade $(33,3 \%)$ e distúrbios musculares $(11,11 \%)$. A Tabela 4 mostra as potenciais interações medicamentosas.

\section{Tabela 4}

Possíveis interações droga-droga entre os medicamentos relatados pelos entrevistados.

\begin{tabular}{clcl}
\hline Droga 1 & Droga 2 & Gravidade* & Efeitos* \\
\hline Ritonavir & Atazanavir & Moderada & Aumento dos efeitos do atazanavir \\
Sinvastatina & Omeprazol & Moderada & $\begin{array}{l}\text { Aumento dos níveis sanguíneos e dos efeitos da } \\
\text { simvastatina }\end{array}$ \\
Sinvastatina & Zidovudina & Moderada & Aumento do risco de distúrbios musculares \\
Sinvastatina & Efavirenz & Moderada & Redução dos efeitos da simvastatina \\
Efavirenz & $\begin{array}{l}\text { Zidovudina / lamivudina } \\
\text { Abacavir }\end{array}$ & Moderada & Aumento do risco de problemas hepáticos \\
Álcool & Mnalapril & Aumento de potássio no sangue \\
Trimetoprim & Atazanavir & Moderada & $\begin{array}{l}\text { Pode alterar os níveis sanguíneos e os efeitos de } \\
\text { ambos os medicamentos }\end{array}$ \\
\hline
\end{tabular}

*Banco de dados eletrônico do Micromedex® Healthcare Series

Fonte: Autores. 
A adesão dos pacientes ao tratamento antirretroviral foi avaliada ao se correlacionar a influência dos dados socioeconômicos sobre essa e utilizando a soma dos itens do questionário, versão validada para a língua portuguesa (Brasil) do CEA-
T-VIH (Tabela 5). Após o somatório do questionário de cada paciente verificou-se que $66,6 \%$ possui uma adesão regular/insuficiente, enquanto 33,3\% possui uma adesão boa (Tabela 6 ), não sendo identificado nenhum paciente com adesão baixa.

\section{Tabela 5}

Descritivos das variações relacionadas a percepção dos pacientes em relação à adesão ao tratamento

\begin{tabular}{|c|c|c|}
\hline Variáveis & Pontuacões possíveis* & Média \\
\hline Deixou de tomar o med. na última semana & 1 sempre a 5 nunca & 054,6 \\
\hline $\begin{array}{l}\text { Deixou de tomar o med. durante un día completo desde } \\
\text { el inicio del tto. durante um dia completo desde o início } \\
\text { do tto }\end{array}$ & $\begin{array}{l}0 \text { no }-1 \text { sí } \\
0 \text { não }-1 \text { sim }\end{array}$ & 0,3 \\
\hline Aderência ao horário & 1 sempre a 5 nunca & 054,3 \\
\hline Sentiu-se melhor como motivo de omissão & 5 sempre a 1 nunca & 4,9 \\
\hline Sentiu-se pior como motivo de omissão & 5 sempre a 1 nunca & 4,6 \\
\hline $\begin{array}{l}\text { Sentiu-se triste ou deprimido como motivo de omissão } \\
\text { Utilización de estrategia }\end{array}$ & $\begin{array}{l}5 \text { siempre a } 1 \text { nunca } \\
5 \text { sempre a } 1 \text { nunca }\end{array}$ & 054,6 \\
\hline Uso de estratégia & 0 não $-1 \mathrm{sim}$ & 000,3 \\
\hline Memória dos medicamentos utilizados & 0 nenhum, 1 metade, 2 tudo & 0,8 \\
\hline Frequência de reforço / encorajamento pelo médico & 1 nunca a 5 sempre & 3,9 \\
\hline $\begin{array}{l}\text { Calidad de la relación médico-paciente } \\
\text { Qualidade do relacionamento médico-paciente }\end{array}$ & 1 ruim a 5 bom & 4,9 \\
\hline Grau de esforço envolvido no tto & 1 muito a 5 nada & 4,3 \\
\hline Percepção de capacidade de continuar com o tto & 1 nada a 5 muito & 044,2 \\
\hline Grau de informação sobre o tto & 1 nada a 5 muito & 4,3 \\
\hline Benefícios que o tto pode supor & 1 nenhum a 5 muitos & 044,3 \\
\hline $\begin{array}{l}\text { ¿Cómo se siente en general desde que ha empezado a } \\
\text { tomar el tto.? } \\
\text { Como você se sente em geral desde que você começou } \\
\text { o tto? }\end{array}$ & 1 muito insatisfeito a 5 muito satisfeito & 4,0 \\
\hline $\begin{array}{l}\text { Intensidad de los efectos secundarios } \\
\text { Intensidade de efeitos colaterais }\end{array}$ & 1 muito intenso a 5 nada intenso & 034,1 \\
\hline Grau de melhoria desde o início do tto & $\begin{array}{l}1 \text { nada a } 5 \text { mucho } \\
1 \text { nada a } 5 \text { muito }\end{array}$ & 044,2 \\
\hline Quanto tempo leva você para continuar com o tto? & $\begin{array}{l}1 \text { mucho tiempo a } 5 \text { poco tiempo } \\
1 \text { tempo longo a } 5 \text { pouco tempo }\end{array}$ & 044,4 \\
\hline $\begin{array}{l}\text { Autovaloración del grado de adhesión } \\
\text { Autoavaliação do grau de adesão }\end{array}$ & 1 nada compatível a 5 muito confiável & 044,0 \\
\hline Dificuldade percebida sobre o tto & 1 dificuldade a 5 nada de dificuldade & 044,2 \\
\hline
\end{tabular}

Legenda: med. $=$ medicamento, tto. $=$ tratamiento, ${ }^{*}$ mayor puntuación mayor grado de adhesión al tratamiento $=$ tratamento, ${ }^{*}$ maior pontuação maior grau de adesão ao tratamento

Fonte: Autores

\section{Tabela 6}

Percentual da classificação em relação a adesão apresentada pelos entrevistados.

\begin{tabular}{lc}
\hline Classificação & Porcentagem \\
\hline Boa & $33,33 \%$ \\
Regular / insuficiente & $66,66 \%$ \\
Baixa & $0 \%$ \\
\hline
\end{tabular}

Fonte: Autores.

\section{DISCUSSÃO}

A adesão é um comportamento multifatorial, dinâmico e variável ao longo do tempo na medida em que está dependente de características do tratamento farmacológico, das características da doença, de fatores psicossociais, da variabilidade individual, da relação terapêutica, e 
dos cuidados de saúde. A identificação das dificuldades enfrentadas relativas ao tratamento, como o tempo de tratamento e o número de comprimidos diários, facilita a reflexão sobre o modo de intervenção ${ }^{8}$.

No presente estudo, a maioria dos indivíduos era do sexo masculino (73,3\%) (Tabela 2). Embora se saiba do fenômeno da feminização ${ }^{9}$ do HIV, os dados corroboram estudos realizados em outras regiões brasileiras, em que predominaram pacientes do sexo masculino ${ }^{10-11}$. Ademais, na última década, observou-se no Brasil um aumento de $67,8 \%$ na taxa de deteç̧ão de casos de AIDS em jovens do sexo masculino e uma redução de $12,2 \%$ entre os do sexo feminino, implicando na progressão de homens vivendo com HIV/AIDS nos próximos anos ${ }^{2}$. Cumpre assinalar que o modelo de masculinidade instituído pela sociedade é um fator que pode influenciar na vulnerabilidade masculina à infecção pelo HIV/AIDS, visto que contribui para que o homem não assuma as mudanças comportamentais necessárias para a prevenção da transmissão do vírus ${ }^{12}$. Contudo, alguns autores apontaram que as mulheres têm faltado ao acompanhamento clínico e esquecido maior número de doses da medicação que os homens e esta diferença seria explicada pelo fato de que as mulheres precisam administrar as rotinas familiares e os cuidados com as crianças, esquecendo-se de si mesmas. Quando são adotadas medidas visando minimizar os encargos das mulheres, há melhora imediata da adesão ${ }^{13}$.

A faixa etária mais prevalente foi acima de 40 anos $(73,3 \%)$ apresentando, portanto, discordância com dados nacionais, no qual se observa uma juvenização do perfil da AIDS. No Brasil, a média de idade de pessoas infectadas é de 35 anos, enquanto neste estudo foi de 44 anos. Essa diferença pode ser explicada pelo fato da predominância de pessoas com mais tempo de diagnóstico presentes em grupos de discussão, não somente em relação ao HIV, mas também de outras doenças crônicas ${ }^{14}$.

A baixa escolaridade dos indivíduos infectados com o HIV/AIDS encontrada demonstra mudança no perfil da epidemia, uma vez que inicialmente a AIDS era mais frequente entre indivíduos do sexo masculino, com melhor poder aquisitivo e maior escolaridade. A escolaridade é um fator capaz de influenciar na realização do tratamento antirretroviral, visto que um nível maior de escolaridade favorece a compreensão sobre a patologia e terapêutica medicamentosa, o que é relevante para a adesão ao tratamento ${ }^{15}$.

É importante ressaltar que a escolaridade apresenta interferência apenas em situações de extrema pobreza, posto que tal condição pode implicar em dificuldade de acesso ao tratamento $^{10}$. Portanto, tal fator não deve ser considerado uma variável associada diretamente a adesão já que se verificou um suporte social, pelos profissionais ali presentes para que os pacientes fossem as reuniões, como programas que provêm serviços ou incentivos como transporte, assistência aos presentes em diversas áreas clinicas, sendo estes alguns aspectos indicados para reforçar o sucesso e manutenção dos pacientes em tratamento clínico. ${ }^{16}$

Os baixos níveis de escolaridade observados neste estudo vão ao encontro da indicação de que se trata de marcadores socioeconômicos e preditores no processo de pauperização da epidemia, característica predominante nas notificações da população com HIV das diversas regiões do Brasil ${ }^{13}$.

Acerca da situação conjugal, observou-se que $86,7 \%$ dos entrevistados eram solteiros. Foi evidenciado em outros estudos que a presença de parceiro no processo do adoecimento repercute de forma positiva no tratamento, melhorando a adesão à TARV ${ }^{17}$.

Verificou-se que muitos pacientes têm resistência em contar o diagnóstico da doença aos seus familiares, o que compromete o apoio familiar que poderia influenciar positivamente no sucesso da terapia. Inclusive, apenas o fato de viver com a família parece tornar os pacientes mais empenhados em seguir o tratamento ${ }^{18}$.

O uso recreacional do álcool foi mencionado por $33,3 \%$ dos entrevistados como uma barreira à adesão. Estes citaram interrupções da tomada de medicamentos prevendo consumo de bebidas alcoólicas e também a utilização da mesma junto com os medicamentos. Diversos estudos destacam o papel do álcool no aumento dos níveis séricos, interferindo no aumento do risco de eventos adversos da terapia ARV, como também elevando o perigo de toxicidades e mesmo possível perda de eficácia dos medicamentos ${ }^{19}$. 
Sobre o tempo de diagnóstico, não é uma variável consensual na literatura em relação a uma maior predição de adesão. Tanto pacientes que se tratavam há menos de seis meses quanto aqueles que se trataram há mais de 10 anos apresentaram níveis de adesão insatisfatórios.

Quando questionados sobre como é o esquema de tratamento e como são os medicamentos utilizados, $20 \%$ demonstraram familiaridade com o esquema terapêutico, citando os nomes dos medicamentos, o horário e como devem ser ingeridos. Embora a memorização dos nomes dos medicamentos constitua um recurso importante para assegurar seu uso correto, a maioria dos pacientes não sabia informar a medicação prescrita. Tal fato pode ser associado à complexidade e diversidade dos nomes dos ARV, o que dificulta sua assimilação ${ }^{20}$.

O número de doses, medicamentos, efeitos colaterais e as mudanças no estilo de vida são fatores que podem levar a uma diminuição na adesão ao tratamento. Geralmente, a TARV é composta de dois ou mais medicamentos. No grupo em estudo, 73,3\% utilizavam dois medicamentos, sendo que $53,3 \%$ dos participantes estavam usandoa combination of 2 NRTIs and an NNRTI. uma combinação de dois ITRN e um ITRNN. This combination of aEssa combinação era uma diretriz do Ministério da Saúde para a terapia inicial contra o HIV. Furthermore, a combination of 2 NRTIs and 2 PIsThe most common combination of 2 NRTIs and an NNRTIA combinação mais comum foi zidovudina/lamivudina com efavirenz $(33,3 \%)^{20}$. Nossos achados foram similares a outros estudos, já que Therapiesterapias involving a combination of more than 4 drugs are used in order toenvolvendo uma combinação de mais de quatro medicamentos são usadas para alcançar o resgate imune ou como recurso para aqueles pacientes que já encontraram algum tipo de resistência ao tratamento ${ }^{21}$. It was found that $55.5 \%$ of seropositive individuals were usingtreatment has already encountered some kind of resistance. Likewise, in the study of Gomes

Entre as IDD identificadas, $44,4 \%$ ocorreram entre os agentes ART usados. A coadministração do ART e outros medicamentos podem resultar em mudanças importantes nos níveis séricos, muitas das quais estão relacionadas a eventos adversos evitáveis. Há evidências de que HARRT, sozinho ou combinado com outros medicamentos, altera o metabolismo CYP450, que foi observado no presente estudo, especialmente nas potenciais interações droga-droga (PIDD) de moderada e superior severidade ${ }^{22}$. A adição de cada terapia de medicamento aumenta o risco de eventos adversos em $10 \%$, incluindo IDD.

Uma parte significante de PIDD pode ser atribuída ao fato de que $100 \%$ do ART neste estudo são metabolizados pelo sistema da enzima CYP450, agindo como substratos (isoenzimas $1 \mathrm{~A} 2$, 2A6, 2B6, 2C19, 2D6 e 3A4), inibidores (isoenzimas $1 \mathrm{~A} 2,2 \mathrm{C} 8 / 9,2 \mathrm{C} 19,2 \mathrm{D} 6,2 \mathrm{E} 1$ e $3 \mathrm{~A} 4)$ e indutores (isoenzimas 1A2, 2B6, 2C8/9 e $3 \mathrm{~A} 4$ ) 3 $^{3}$. Teoricamente, a coadministração de inibidores ou indutores pode causar mudanças clinicamente significantes em farmacocinética e farmacodinâmica de outros medicamentos (substratos). No entanto, a taxa e extensão do metabolismo são dependentes na expressão dessas isoenzimas, que podem ser influenciadas por polimorfismos genéticos. Estima-se que a genética pode explicar $20-95 \%$ da variabilidade em resultados terapêuticos e tóxicos, incluindo IDD. Na avaliação de um IDD específico é importante perceber o potencial inibitório relativo da droga para a enzima em particular ${ }^{5}$.

Potenciais interações medicamentosas podem ter sido subestimadas devido à falta de informações sobre comorbidades, infecções oportunistas, automedicação sem prescrição médica, modo prático e o uso de plantas medicinais. Embora o estudo não tenha sido desenhado para investigar o impacto clínico da IDD, as descobertas, ainda que limitadas, são relevantes para pacientes com HIV, especialmente por apontar os grupos mais vulneráveis à IDD. Adicionalmente, os regimes terapêuticos usados em pacientes com HIV são bastante similares no mundo todo, principalmente depois do advento do HAART. Geralmente, a prescrição destas drogas é de responsabilidade do infectologista, um aspecto que reduz a variabilidade da prática clínica ${ }^{5}$.

A adesão dos pacientes ao tratamento antirretroviral foi avaliada utilizando a soma dos itens do questionário, versão validada para a língua portuguesa (Brasil) do CEAT-VIH. O questionário CEAT-VIH permitiu observar a percepção do paciente em relação ao tratamento e de como 
este interfere em sua vida diária, interfere significativamente na adesão, como demonstrado nas questões sobre sentir-se pior, sentir-se melhor ou sentir-se triste/deprimido, assim, as questões individuais revelam-se como preponderantes no comportamento de adesão ${ }^{23}$.

Uma boa compreensão do tratamento também aparece como fator importante nesse aspecto, isto está evidenciado em nosso estudo na questão sobre lembrar-se ou não dos medicamentos, aqueles que não se lembravam ou se lembravam somente de um dos medicamentos que tomavam tiveram uma maior probabilidade de apresentar uma adesão insatisfatória. Em relação ao serviço de saúde, sentir-se animado e motivado pelo médico quando apresentava bons resultados de exames, esteve associado a uma boa adesão. Este resultado corrobora os de outros estudos que apontaram a relação com a equipe de saúde como fator determinante no processo de adesão. Além disso, o consumo de álcool esteve associado a não adesão quando mensurada pelo autorrelato da perda de doses, mas não quando mensurada pelo CEAT-VIH. Em relação ao uso ou abuso de álcool como fator de risco para não adesão, não existe consenso na literatura, contudo, neste estudo, todos que revelaram tal associação apresentaram adesão insatisfatória. É possível que o abuso de álcool reflita uma menor habilidade de autocuidado pelo paciente, também demonstrada no comportamento de não adesão a TARV ${ }^{24}$.

Na associação da adesão com o número de comprimidos tomados ao dia, a utilização de esquemas terapêuticos simples e adequados considerando o perfil biopsicossocial do indivíduo, assim como as suas rotinas, tem grande significância para minimizar a adesão inadequada. Outro aspecto importante que tende a favorecer a adesão adequada é a simplificação e adequação do esquema escolhido, a rotina de cada um, sempre que possível. O número total de comprimidos ingeridos pode aumentar a taxa de adesão inadequada em $12 \%$ a cada 10 comprimidos. Sobre a posologia, esta variou de uma a duas vezes ao dia, prevalecendo pacientes que precisam tomar as medicações duas vezes ao dia $(53,3 \%)^{15}$.

As dificuldades de adesão em HIV/AIDS decorrem, em parte, da complexidade da TARV, na medida em que alguns medicamentos precisam ser ingeridos com alimentos, outros em jejum, ou em sequências temporais combinadas com outros medicamentos, o que exige organização e compromisso do paciente em relação ao seu tratamento ${ }^{25}$. Nenhum dos entrevistados relatou efeitos colaterais mais intensos e maior dificuldade em tomar os medicamentos, respostas que se correlacionam com maior probabilidade de serem não aderentes ao tratamento.

É importante observar as discrepâncias apresentadas entre a percepção dos pacientes em relação à adesão, que apresentou uma média de 75,2 (Tabela 6), em contraste a porcentagem de adesões regulares, as quais apresentaram uma porcentagem de $66,66 \%$. Estes dados demonstram que os entrevistados acreditam ter uma boa adesão ao tratamento, embora, através da classificação realizada com base no CEAT-HIV, ter sido demostrado que a maioria não está dentro do padrão de boa adesão. Uma maior investigação sobre os fatos ocasionadores dessa discrepância pode ser realizada pelo farmacêutico, já que este é o profissional que reúne melhores condições para orientar o paciente sobre o uso correto dos medicamentos, esclarecendo dúvidas e favorecendo a adesão e sucesso do tratamento. Portanto, cabe assinalar a importância do farmacêutico como participante fixo na equipe de saúde que interage diretamente com pessoas que convivem com HIV/AIDS.

O que se verifica, na prática, é que diferentes estratégias podem ser utilizadas para promover a adesão, contudo, o importante é que o desenvolvimento e a implementação dessas intervenções sejam realisticamente desenhados para grupos específicos, considerando as características individuais, o estilo de vida e o suporte social.

\section{CONCLUSÕES}

Esse estudo permitiu avaliar a adesão das pessoas convivendo com HIV/AIDS ao tratamento antirretroviral no Grupo de Adesão do IDTNP, no qual foi constatada como insatisfatória na população estudada, em decorrência de fatores biopsicossociais que os participantes estão inseridos.

No contexto da farmacoepidemiologia antirretroviral, as potenciais interações medicamento- 
sas identificadas neste estudo de severidade moderada e superior foram eventos que podem levar a toxicidade devido aumento da concentração de um dos fármacos na corrente sanguínea ou ainda ocasionar o desenvolvimento de danos hepáticos.

A discrepância entre a percepção sobre a adesão e a real adesão dos pacientes demonstra a importância do farmacêutico como participante efetivo da equipe multiprofissional que interage com pessoas que convivem com HIV/AIDS, sendo este o profissional capacitado para orientar, educar e instruir o paciente sobre todos os aspectos relacionados ao medicamento.

Por esta razão, julga-se necessário uma maior abordagem nas reuniões dos preditores citados nesta pesquisa, assim como fortalecimento da participação do farmacêutico que acompanha as pessoas que convivem com HIV/AIDS.

\section{REFERÊNCIAS}

1. Fraporti C. Características sociodemográficas da população que busca o teste rápido como diagnóstico inicial do HIV em uma Unidade de Pronto-Atendimento. Canoas. Dissertação [Mestrado em Saúde e Desenvolvimento Humano] - Centro Universitário La Salle; 2015

2. Ministério da Saúde (Brasil). Manual técnico para diagnóstico de infecção pelo HIV. 2. Ed. Brasília:, Secretaria de Vigilância e saúde. Departamento de DST, AIDS e Hepatites Virais; 2014.

3. Bae JW, Guyer W, Grimm K, Altice FL. Medication persistence in the treatment of HIV infection: a review of the literature and implications for future clinical care and research. AIDS [internet]. 2011 [acesso em 13 ago 2019];25(3):279-90. Disponível em: https://www.ncbi. nlm.nih.gov/pubmed/21239892

4. Rosenbloom DIS, Hill AL, Rabi SA, Siliciano RF, Nowak MA. Antiretroviral dynamics determines HIV evolution and predicts therapy outcome. Nat med [internet]. 2012 [acesso em 13 ago 2019];18(9):1378-85. Disponível em: https://www.nature.com/articles/nm.2892.pdf

5. Santos WM, Secoli SR, Padoin, SMM. Potential drug interactions in patients given antiretroviral therapy. Rev Latino-Am Enferm [internet]. 2016 [acesso em 13 ago 2019];24:e2832. Disponivel em: http://www.scielo.br/ pdf/rlae/v24/0104-1169-rlae-24-02832.pdf

6. Remor E, Milner-Moskovics J, Preussler, G. Adaptação brasileira do "Cuestionario para la Evaluación de la Adhesión al Tratamiento Antiretroviral". Rev saude publica [internet]. 2007 [acesso em 13 ago 2019];41(5):685-94. Disponível em: http://www.scielo.br/scielo.php?pid=S0034$89102006005000043 \&$ script=sci_abstract\&tlng=pt
7. Micromedex ${ }^{\circledR}$ Healthcare Series [Internet database]. Greenwood Village, Colo: Thomson Healthcare. Atualizado periodicamente.

8. Fiuza MLT, Lopes EM, Alexandre HO, Dantas PB, Galvão MTG, Pinheiro AKB. Adesão ao tratamento antirretroviral: assistência integral baseada no modelo de atenção às condições crônicas. Esc Anna Nery [internet]. 2013 [acesso em 13 ago 2019];17(4):740-8. Disponível em: http://www. scielo.br/pdf/ean/v17n4/1414-8145-ean-17-04-0740.pdf

9. Reis RK, Santos CB, Dantas RAS, Gir E. Qualidade de vida, aspectos sociodemográficos e de sexualidade de pessoas vivendo com HIV/AIDS. Texto \& contexto enferm [internet]. 2010 [acesso em 13 ago 2019];20(3):565-75. Disponível em: http://www.scielo.br/pdf/tce/v20n3/19.pdf

10. Foresto JS, Melo ES, Costa CRB, Antonini M, Gir E, Reis RK. Adesão à terapêutica antirretroviral de pessoas vivendo com HIV/aids em um município do interior paulista. Rev Gaúcha Enferm [internet]. 2017 [acesso em 13 ago 2019];38(1):e63158. Disponível em: http://www.scielo.br/pdf/rgenf/v38n1/0102-6933-rgenf-1983-144720170163158.pdf

11. Souza CC, Mata LRF, Azevedo C, Gomes CRG, Cruz GECP, Toffano SEM. Interiorização do HIV/AIDS no brasil: um estudo epidemiológico. Rev bras ciênc saúde [internet]. 2013 [acesso em 13 ago 2019];11(35):25-30. Disponível em: https://seer.uscs.edu.br/index.php/revista_ciencias_saude/article/view/1798/1380

12. Moraes DCA, Oliveira RC, Costa SFG. Adesão de homens vivendo com HIV/Aids ao tratamento antirretroviral. Esc Anna Nery [internet]. 2014 [acesso em 13 ago 2019];18(4):676-81. Disponível em: http://www.scielo.br/ pdf/ean/v18n4/1414-8145-ean-18-04-0676.pdf

13. Colombrini MRC, Lopes MHBM, Figueiredo RM. Adesão à terapia antiretroviral para HIV/AIDS. Rev esc enferm USP [online]. 2006 [acesso em 13 ago 2019];40(4):576-81. Disponível em: http://www.scielo.br/pdf/reeusp/v40n4/ v40n4a17.pdf

14. Ministério da Saúde (Brasil) [Homepage na internet]. Boletim Epidemiológico de AIDS/DST. Brasília: Ministério da Saúde; 2013.[Acesso em: 21 de out. 2017]. Disponível em :http://www.aids.gov.br/pt-br/pub/2016/ boletim-epidemiologico-de-aids-2016

15. Silva ALCN, Waidman MAP, Marcon SS. Adesão e não-adesão à terapia anti-retroviral: as duas faces de uma mesma vivência. Rev Bras enferm [online]. 2009 [acesso em 13 ago 2019];62(2):213-20. Disponível em: http://www.scielo.br/pdf/reben/v62n2/a07v62n2.pdf

16. Lemos LA, Fiuza MLT, Reis RK, Ferrer AC, Gir E, Galvão MTG. Adherence to antiretrovirals in people coinfected with the human immunodeficiency virus and tuberculosis. Rev Latino-Am Enferm [internet]. 2016 [acesso em 18 jul 2017];24:e2691. Disponível em: http://www. scielo.br/pdf/rlae/v24/0104-1169-rlae-24-02691.pdf

17. Ferreira BE, Oliveira IM, Paniago AMM. Qualidade de vida de portadores de HIV/AIDS e sua relação com linfócitos $\mathrm{CD} 4+$, carga viral e tempo de diagnóstico. Rev Bras epidemiol [internet]. 2012 [acesso em 13 ago 
2019];15(1):75-84. Disponível em: http://www.scielo. br/pdf/rbepid/v15n1/07.pdf

18. Blatt CR, Citadin CB, Souza FG, Mello RS, Galato D. Avaliação da adesão aos anti-retrovirais em um município no Sul do Brasil. Rev Soc Bras Med. Trop [internet]. 2009 [acesso em 13 ago 2019];42(2):131-6. Disponível em: http://www.scielo.br/pdf/rsbmt/v42n2/v42n2a07. pdf

19. Sousa Filho MP, Luna IT, Silva KL, Pinheiro PNC. Pacientes vivendo com HIV/AIDS e coinfecção tuberculose: dificuldades associadas à adesão ou ao abandono do tratamento. Rev Gaúcha Enferm [internet]. 2012 [acesso em 13 ago 2019];33(2):139-45. Disponível em: http:// www.scielo.br/pdf/rgenf/v33n2/20.pdf

20. Ministério da Saúde (Brasil). Programa Nacional de DST e AIDS. Recomendações para a terapia antirretroviral em adultos infectados pelo HIV. 7 th ed.7th ed. Brasília: Secretaria Programa Nacional de DST e AIDS. Recomendações para a Brasília: Secretaria de Vigilância em Saúde; 2008.

21. Gomes RRFM, Machado CJ, Acurcio FA, Guimarães MDC. Utilização dos registros de Utilização dos registros de dispensação da farmácia como indicador da não-adesão à terapia antirretroviral em indivíduos infectados pelo HIV. Cad Saude Publica 2009; Cad Saúde Públi- ca [internet] 2009 [acesso em 13 ago 2019];25:495506.25(3):495-506. Disponível em: http://www.scielo. $\mathrm{br} / \mathrm{pdf} / \mathrm{csp} / \mathrm{v} 25 \mathrm{n} 3 / 04 . \mathrm{pdf}$

22. Fonseca LC, Martins FJ, Vieira RCPA, Pereira RMC, Ferreira AS, Raposo NRB. Evaluation of inadequate anti-retroviral treatment in patients with HIV/AIDS. Rev Soc Bras Med Trop [internet]. 2012 [acesso em 13 ago 2019];45(2):151-5. Disponível em: http://www.scielo. $\mathrm{br} / \mathrm{pdf} / \mathrm{rsbmt} / \mathrm{v} 45 \mathrm{n} 2 / \mathrm{v} 45 \mathrm{n} 2 \mathrm{a} 02 . \mathrm{pdf}$

23. Campos LN, Guimaraes MDC, Remien RH. Anxiety and depression symptoms as risk factors for non-adherence to antiretroviral therapy in Brazil. AIDS Behav [internet]. 2010 [acesso em 13 ago 2019];14(2):289-99. Disponível em: https://link.springer.com/content/pdf/10.1007\%2Fs10461-008-9435-8.pdf

24. Ceccato MGB, Acurcio FA, Cesar CC, Bonolo PF, Guimarães MDC. Compreensão da terapia anti-retroviral: uma aplicação de modelo de traço latente. Cad Saúde Pública [internet]. 2008 [acesso em 13 ago 2019];24(7):168998. Disponível em: http://www.scielo.br/pdf/csp/ v24n7/23.pdf

25. Irffi G, Soares RB, Souza SA. Fatores socioeconômicos, demográficos, regionais e comportamentais que influenciam no conhecimento sobre HIV/AIDS. Rev Econ. $2010 ; 11(2): 333-56$. 